\title{
Carcinoma Mucoepidermoide de Glándulas Salivales: Factores Pronósticos Histológicos e Inmunohistoquímicos
}

\author{
Mucoepidermoid Carcinoma of Salivary Glands: \\ Histological and Inmunohistochemical Prognostic Factors
}

Rodolfo Esteban Avila; María Elena Samar; Ismael Fonseca; Luis Olmedo; Onell Gabriela Asís \& Ricardo Ferraris

AVILA, R. E.; SAMAR, M. E.; FONSECA, I.; OLMEDO, L.; ASÍS, O. G. \& FERRARIS, R. Carcinoma mucoepidermoide de glándulas salivales: factores pronósticos histológicos e inmunohistoquímicos. Int. J. Morphol., 29(2):455-462, 2011.

RESUMEN: Los tumores de las glándulas salivales representan entre el 2 y $3 \%$ de las neoplasias de cabeza y cuello. El carcinoma mucoepidermoide (CME) es el tumor maligno más común en las glándulas salivales; su comportamiento biológico se relaciona con el grado histológico tumoral (bajo, intermedio y alto). Está formado por células mucosas, epidermoides, intermedias, columnares y claras. El objetivo del presente trabajo fue presentar la casuística de CME de nuestro laboratorio en el período 1999-2009 para determinar grado histológico y potencial proliferativo como factores pronósticos ya que éstos son importantes para guiar el tratamiento y diseñar recomendaciones para los pacientes. Cortes seriados de 13 casos de CME se colorearon con H/E, Masson, PAS, Dane/Alcian blue y Azul de toluidina. Se realizó inmunomarcación para ki67. Se analizó su grado histológico según el sistema de gradación tumoral de Auclair et al., que evalúa variables observables con microscopio óptico. 8 tumores se localizaron en parótida, 3 en paladar, 1 en submandibular y 1 en glándulas genianas. Histológicamente 3 casos fueron de alto grado, 2 de grado intermedio y 8 de bajo grado, según sus características citológicas, patrón de invasión y tipo celular. En dos casos de bajo grado se observaron cristales de colesterol, macrófagos y células gigantes de cuerpo extraño. La expresión de ki67 fue importante en tumores de alto grado. La parótida fue la más afectada seguida por paladar y submaxilar, lo que acuerda con la bibliografía. El grado histológico tumoral se asoció con la expresión de Ki 67, factores pronósticos relevantes en el CME, que deben asociarse a estudios cínicos y análisis de supervivencia. Continuaremos el presente trabajo con la realización de estudios de los residuos azúcares terminales y subterminales de los carbohidratos con lectinhistoquímica, para detectar modificaciones sutiles de la glicosilación de las proteínas celulares, rasgo común en el proceso de transformación neoplásica.

PALABRAS CLAVE: Glándulas salivales; Carcinoma mucoepidermoide; Factores pronósticos.

\section{INTRODUCCIÓN}

Las neoplasias de glándulas salivales mayores y menores humanas son un desafío tanto para clínicos como para histopatólogos, ya que son entidades nosológicas poco frecuentes y sus características histológicas, clínicas, epidemiológicas y evolutivas son múltiples y variadas (Speight \& Barret, 2002; Licitra et al., 2003; Pérez Ordoñez, 2003; Lang et al., 2005; Ascani et al., 2006; Leivo, 2006; Samar et al., 2009).

Robbins (Kumar et al., 2005) menciona que en las glándulas salivales se originan no menos de 30 tipos histológicos diferentes de neoplasias. Estas representan menos del $2 \%$ de todas las neoplasias humanas y alrededor del 3\% de los tumores de cabeza y cuello (Kumar et al.; Védrine et al., 2006; Ito et al., 2009).
La clasificación de estos tumores así como su diversidad morfológica son factores complejos cuya precisión es de importancia fundamental a la hora de un diagnóstico preciso y un tratamiento adecuado.

Según la teoría bicelular, los tumores de glándulas salivales están formados por: a- células luminales ductales y/o células acinares más células mioepiteliales, b- o células ductales o acinares, c- o células mioepiteliales, exclusivamente (Allon et al., 2006).

A este patrón celular se agrega la producción de matriz extracelular por parte de las células mioepiteliales neoplásicas, colágeno, fibras elásticas, glicoproteínas, glucosaminoglucanos y proteoglicanos (Dardick, 1991). 
Entre el 70 al $80 \%$ de las neoplasias asienta en parótida, mientras que el paladar es el sitio más común para glándulas salivales menores (Ellis \& Auclair, 2008).

Entre los tumores benignos el más frecuente es el adenoma pleomorfo, en tanto que el carcinoma mucoepidermoide (CME) representa el tumor maligno más frecuente según el Instituto de Patología de las Fuerzas Armadas de Washington-AFIP- (Ellis \& Auclair).

El carcinoma mucoepidermoide es un tumor epitelial maligno formado por una proporción variable de células mucosas, epidermoides, intermedias, columnares y claras, que presenta frecuentemente un componente quístico. Su comportamiento biológico se relaciona con el grado histológico tumoral -CME de bajo, intermedio y alto grado(Luna, 2006; Ozawa, 2008; Ellis \& Auclair).

El objetivo del presente trabajo es presentar la casuística de CME de nuestro laboratorio en el período 19992009 para determinar grado histológico y potencial proliferativo como factores pronósticos ya que éstos son importantes para guiar el tratamiento y diseñar recomendaciones para los pacientes (Loh et al., 2009).

\section{MATERIAL Y MÉTODO}

Se estudiaron cortes histológicos de 13 casos de CME provenientes de cirugías de clínicas privadas y hospitales públicos de la ciudad de Córdoba, los que fueron procesados según la siguiente metodología:

- Estudio estructural e histoquímico convencional: Las muestras se procesaron según la técnica histológica de rutina. Se realizaron cortes seriados (de $5 \mu \mathrm{m}$ ) de cada muestra, donde se aplicaron las siguientes técnicas: * Hematoxilina y eosina, * Tricrómico de Masson, *, *Tricrómico de Dane, PAS (para glucoproteínas), *Alcian blue a pH 2,5 y 1,0 (para

Tabla I. Graduación Tumoral (Auclair et al., 1992)

\begin{tabular}{lc}
\hline Parámetros & Puntaje \\
Componente intraquístico $<20 \%$ & +2 \\
Invasión neural & +2 \\
Necrosis & +3 \\
4 mitosis/10 campos a mayor aumento & +3 \\
Anaplasia & +4 \\
Grado & \\
Bajo & \\
Intermedio & $0-4$ \\
Alto & $5-6$ \\
\hline
\end{tabular}

carboximucinas y sulfomucinas), *Alcian blue/sialidasa, *Azul de toluidina a pH 3,8 (para sustancias basófilas y metacromáticas) (Samar et al., 2004).

- Estudio inmunohistoquímico: Marcación con ki67 de las células incluidas en el ciclo celular. La técnica se realizó en cortes de material incluido en parafina de $4 \mathrm{~mm}$ de espesor, con kit LSAB+ de DAKO. La evaluación cualitativa de la marcación se realizó en términos de intensidad de coloración (Samar et al., 2004).

Se analizó su grado histológico según el sistema de gradación tumoral de Auclair et al. (1992), que evalúa variables observables con microscopía óptica (Tabla I).

\section{RESULTADOS}

La apariencia histológica del tumor dependía principalmente del subtipo histológico y de su grado de diferenciación.

Los CEM de bajo grado de malignidad presentaron un predominio de áreas quísticas relacionadas con células mucosecretoras bien diferenciadas (Fig. 1). Tanto las células mucosas, el tipo celular predominante, como el contenido quístico y el mucus que invadía el estroma eran fuertemente PAS reactivos, alcianófilos a ambos $\mathrm{pH}$ y metacromáticos alcohol resistentes (Figs. 2 a y b; 3). La acción de la sialidasa disminuyó notablemente la alcianofilia. Se observaron además, áreas sólidas con células epidermoides y claras entre las que aparecían células mucosas dispersas (Figs. 4a y b). La atipia celular era mínima.

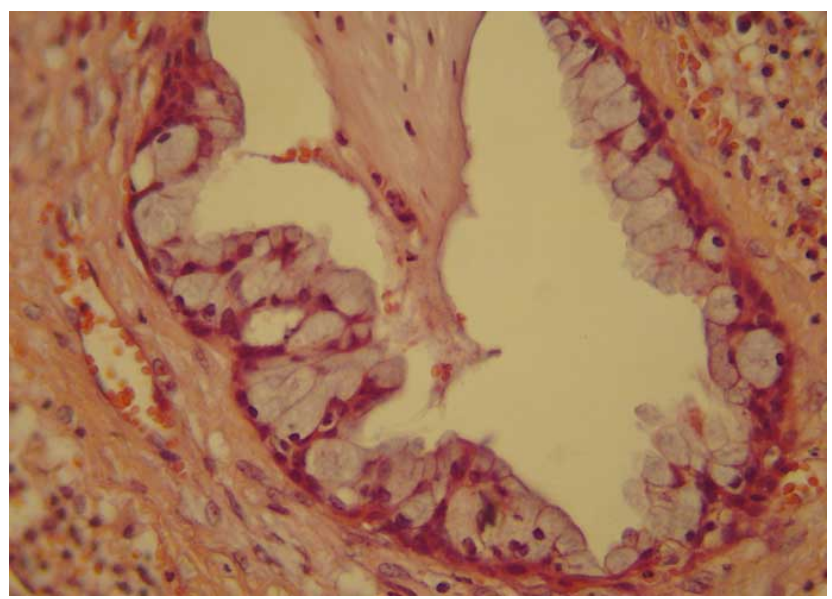

Fig. 1. CME de bajo grado.Area quística con células mucosecretoras. H/E. 200x. 

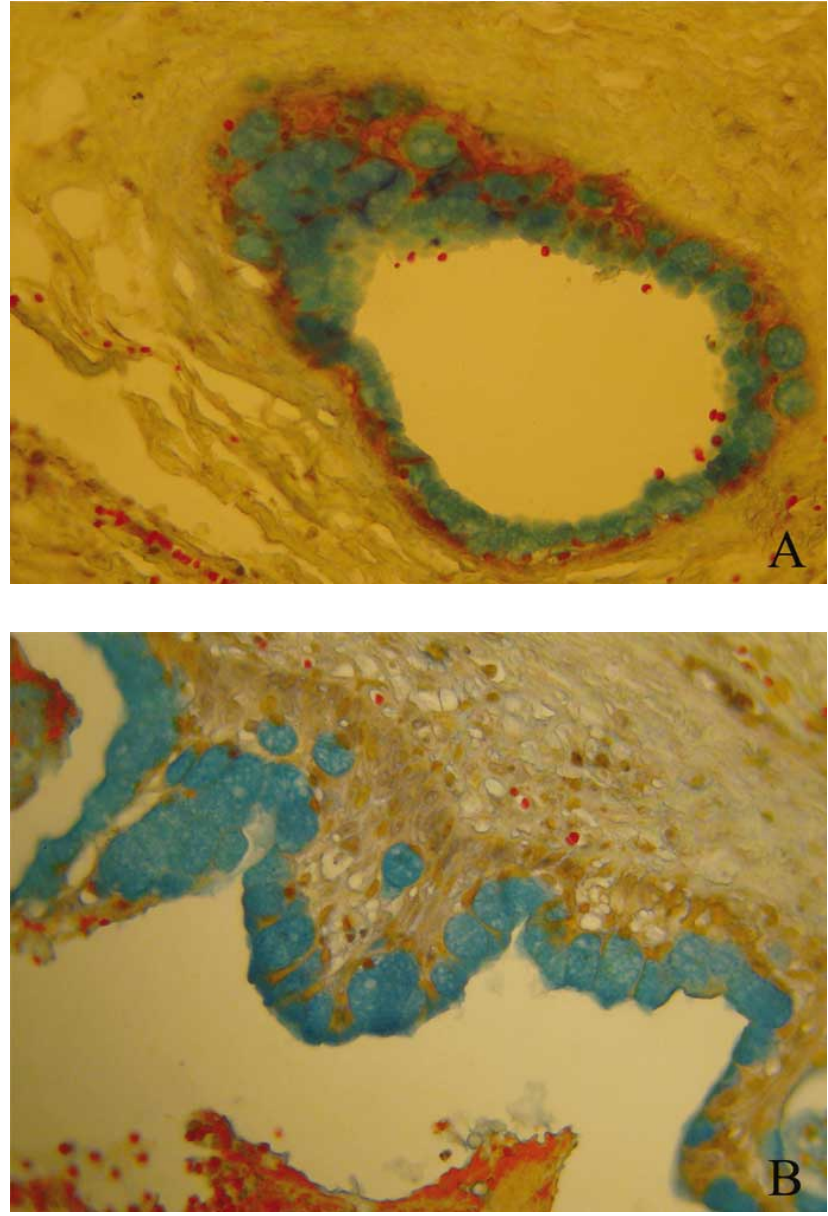

Fig. 2. CME de bajo grado.Quistes con células mucosas alcianófilas. Tricrómico de Dane/Alcian blue. A: 100x. B: 450x.

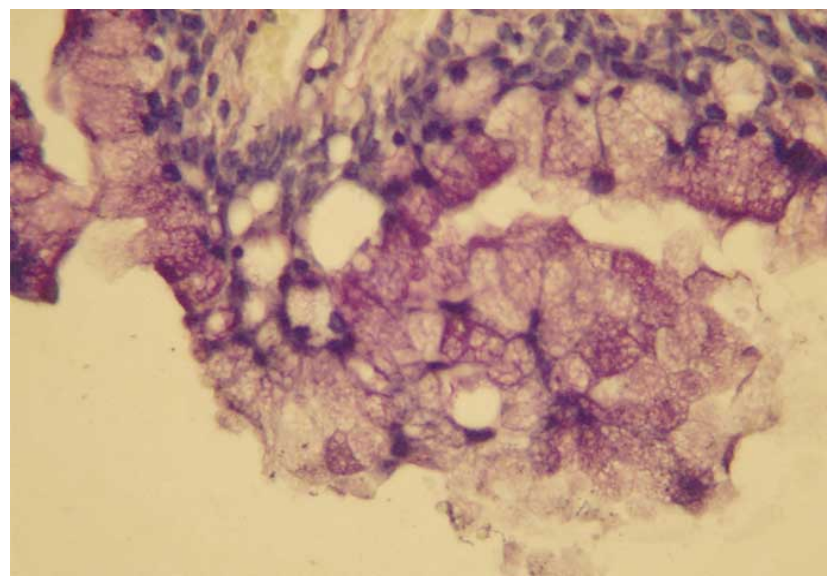

Fig. 3. CME de bajo grado. Estructura quística con células secretoras de mucus intensamente metacromático. Azul de toluidina $\mathrm{pH}$ 3.8. $450 \mathrm{X}$.

En dos casos de CME de bajo grado tumoral (score de 0 a 4), localizados en glándulas genianas y paladar, se observaron focos hemorrágicos y cristales de colesterol asociados a macrófagos, lipófagos y hemosiderófagos (Figs. 5 a y b).
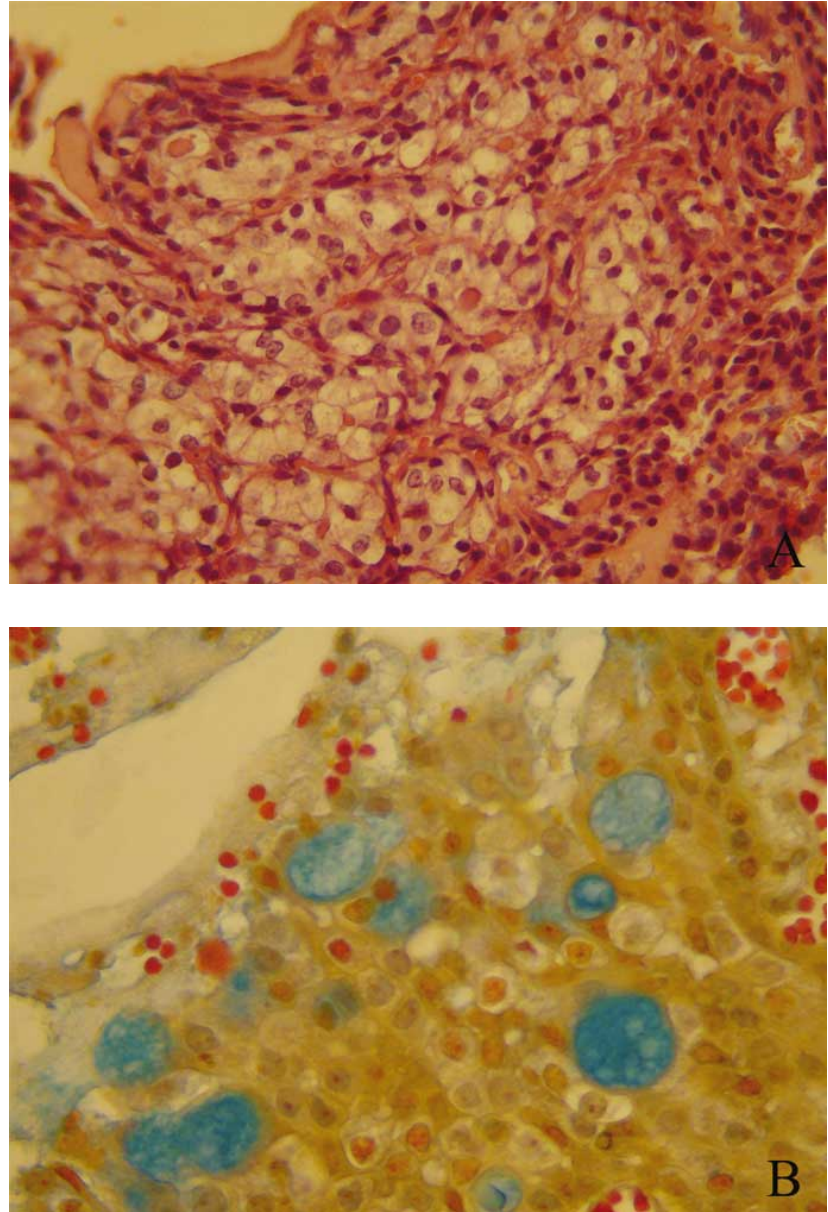

Fig. 4. CME de bajo grado. A. Nido de células claras y epidermoides. H/E. 200x. B. Nido de células claras, epidermoides y mucosas. Vasos sanguíneos dilatados. Tricrómico de Dane/Alcian blue. 450x.

La presencia de los cristales de colesterol se asoció a una marcada reacción a cuerpo extraño con células gigantes, las que se localizaban vecinas a dichos cristales. (Fig. 6).

En los CME de grado intermedio el contenido de mucinas era menor en relación a los CME de bajo grado, mientras que los espacios quísticos eran menos prominentes. Los tipos celulares fueron semejantes a los tumores de bajo grado, con predominio de células intermedias con atipias aisladas.

Los cortes histológicos correspondientes a pacientes con tumores de alto grado presentaron glándulas infiltradas por una neoplasia maligna constituida por áreas sólidas con células anaplásicas acidófilas, con pleomorfismo celular y nuclear (anisocitosis y anisocariosis), macrocariosis, hipercromatismo nuclear, envolturas nucleares irregulares, cromatina en grandes grumos, nucléolos prominentes y mitosis atípicas fáciles de observar (Figs. 7a y b). 

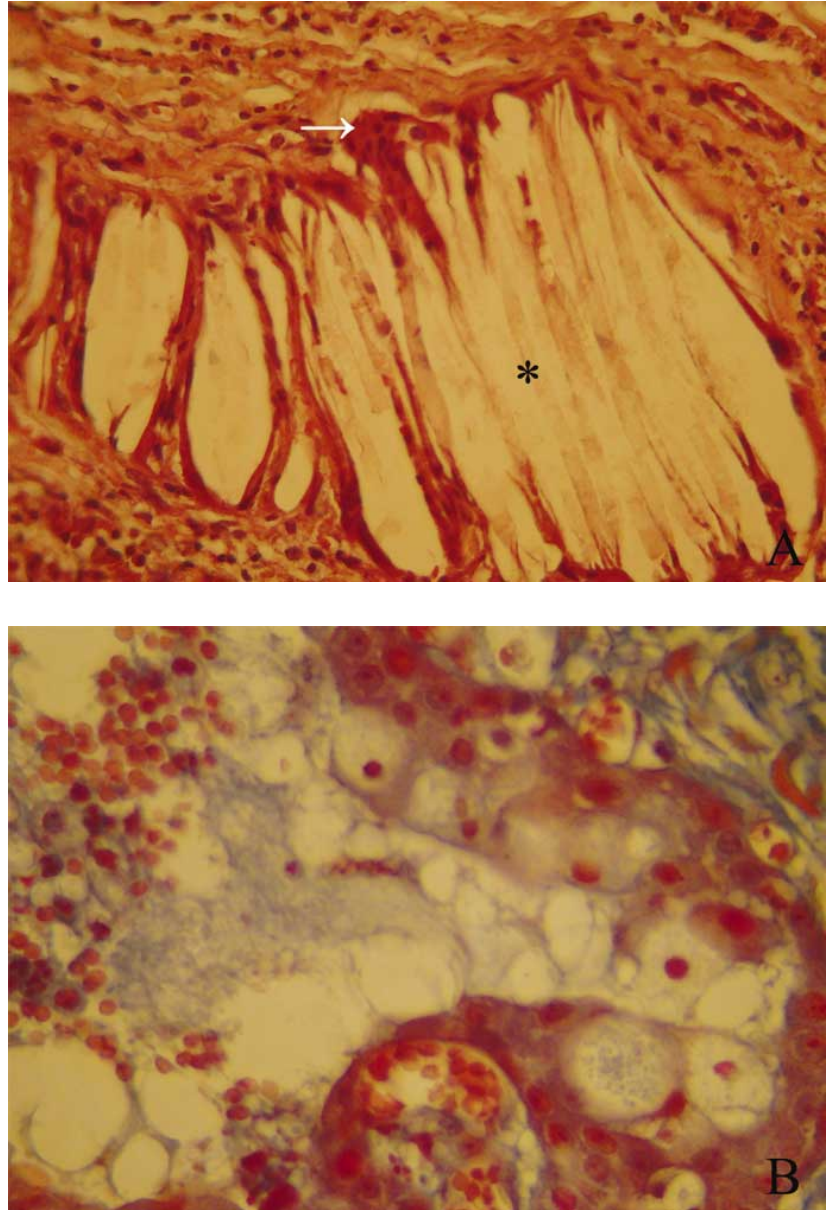

Fig. 5. CME de bajo grado. A. Cristales de colesterol (asterisco). Célula gigante de cuerpo extraño (flecha). Tricrómico de Masson. 100x. B. Nido de macrófagos. Tricrómico de Masson. 450x.

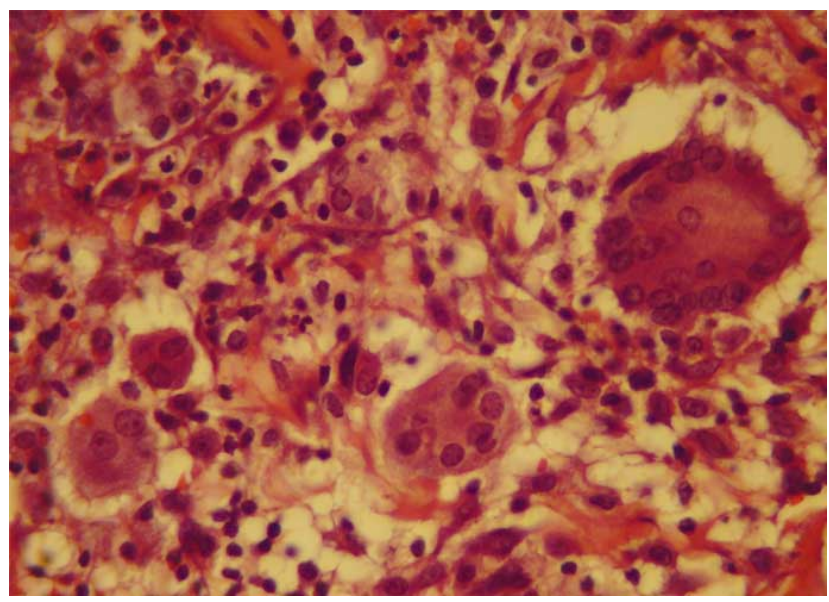

Fig. 6. CME de bajo grado. Células gigantes de cuerpo extraño. H/ E. 450x.

Se observó invasión de tejidos blandos, perineural y vascular.
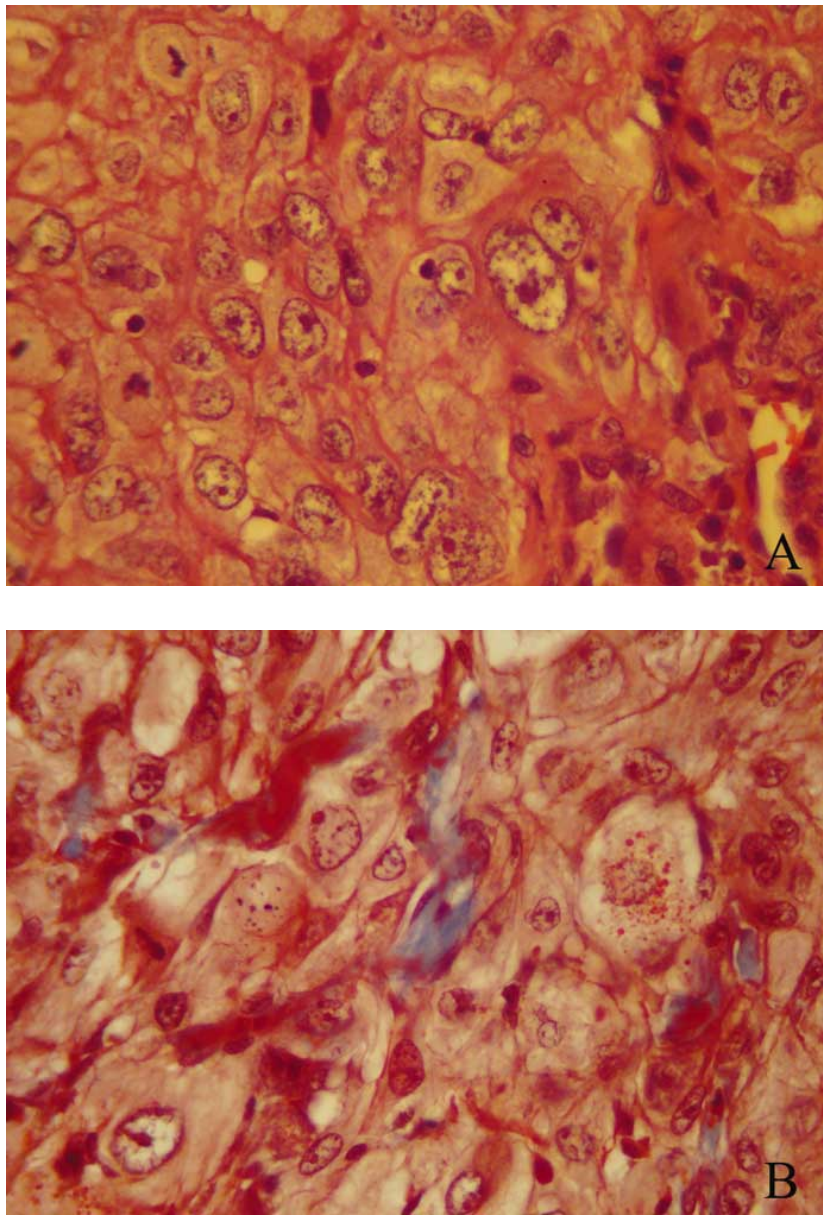

Fig. 7. CME de alto grado. Células anaplásicas. A. H/E. 450x. B. Tricrómico de Masson. 450x.

Las células mucosecretoras eran muy escasas y difíciles de identificar. Se encontraron además células claras PAS positivas, con nucléolos evidentes. Algunas células tumorales se disponían en túbulos con necrosis central rodeados de estroma hialinizado e infiltrado inflamatorio.

La expresión de Ki 67 fue importante en tumores de alto grado (score de 7 o más), (Fig 8 A, B y C).

Ocho tumores se localizaron en parótida, 3 en paladar, 1 en submandibular y 1 en glándulas genianas. Histológicamente 3 casos fueron de alto grado, 2 de grado intermedio y 8 de bajo grado, según sus características citológicas,patrón de invasión y tipo celular.

En la Tabla II se sintetizan las características epidemiológicas e histológicas de los 13 casos estudiados. 

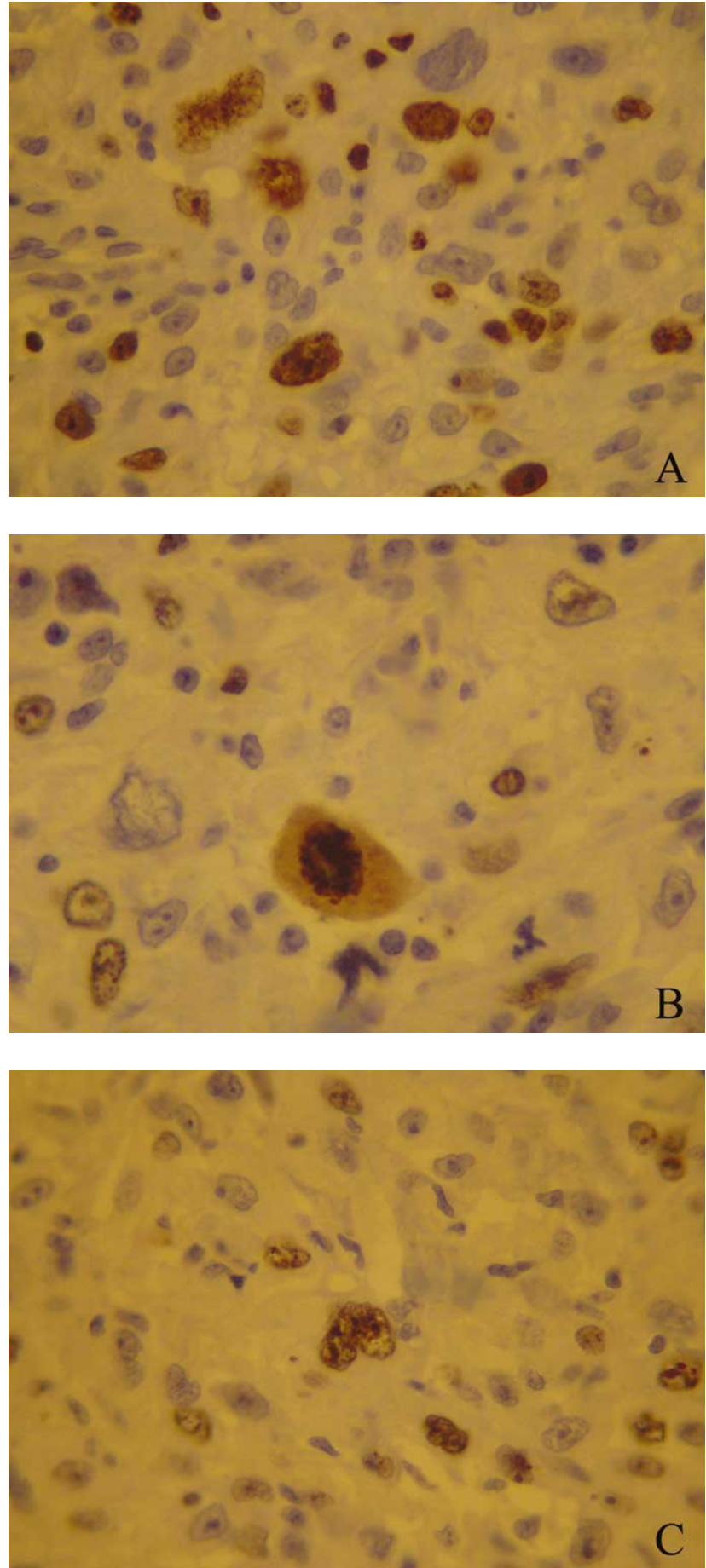

Fig. 8. CME de alto grado. A, B y C: Inmunomarcación ki67 positiva. 450x.

\section{DISCUSIÓN}

El CME es un tumor maligno que afecta en un 53 a $60 \%$ a las glándulas salivales mayores y un $35 \%$ a las glándu-
Tabla II. Características epidemiológicas e histológicas de la casusística de carcinoma mucoepidermoide.

\begin{tabular}{|c|c|c|c|}
\hline Localización & $\begin{array}{l}\text { Edad } \\
\text { (años) }\end{array}$ & Sexo & $\begin{array}{c}\text { Grado } \\
\text { histológico }\end{array}$ \\
\hline Parótida & 70 & $\mathrm{~F}$ & Alto \\
\hline Parótida & 50 & $\mathrm{~F}$ & $\begin{array}{c}\text { Bajo } \\
\text { (recurrencia) }\end{array}$ \\
\hline Parótida & 61 & M & Intermedio \\
\hline Parótida & 21 & M & Bajo \\
\hline Parótida & 15 & $\mathrm{~F}$ & Alto \\
\hline Parótida & 21 & $\mathrm{~F}$ & Bajo \\
\hline Parótida & 44 & $\mathrm{~F}$ & Bajo \\
\hline Parótida & 56 & M & Alto \\
\hline Submandibular & 62 & $\mathrm{~F}$ & Bajo \\
\hline Paladar & 45 & M & Intermedio \\
\hline Paladar & 16 & $\mathrm{~F}$ & Bajo \\
\hline Paladar & 37 & $\mathrm{~F}$ & Bajo \\
\hline Glándulas genianas & 40 & $\mathrm{~F}$ & Bajo \\
\hline
\end{tabular}

las salivales accesorias (Luna; Ellis \& Auclair; Robinson, 2010). Fue estudiado y presentado por primera vez por Stewart et al. (1945) (apud Shafer \& Levy, 2001).

La glándula parótida es el sitio más frecuente, $40 \%$ de los casos, con un $11 \%$ aproximadamente para submandibular y $1 \%$ para sublingual (Luna).

En nuestra casuística, la parótida fue la localización más frecuente de CME, seguida por paladar y submandibular, lo que acuerda con la literatura. Las mujeres fueron las más afectadas, dato que coincide con Ellis \& Auclair, quienes mencionan que afecta en un $62 \%$ a las mujeres, como ocurre con los tumores de glándulas salivales en general.

La relación entre el sitio primario del CME y su pronóstico es controversial. Se ha descrito que las diferencias en el sitio primario no afectan la sobrevida de los pacientes. Sin embargo, algunos autores reportaron que la localización del tumor en glándula submandibular tiene peor pronóstico (Ozawa). Por lo tanto, para dilucidar este aspecto se necesitan estudios con un número significativo de casos.

El grado histológico tumoral se asoció con la expresión de ki67. La proliferación celular es uno de los me- 
canismos biológicos más importantes en la oncogénesis. Gran número de trabajos han demostrado que la actividad proliferativa tiene un gran significado pronóstico en varios tipos de cáncer. El marcador ki67, empleado por nosotros, permite evaluar la fracción de crecimiento, proporción de células comprometidas en el ciclo celular (Pich et al., 2004).

Existe una importante variación en relación al tipo, distribución y patrón de crecimiento de las células en este tumor (Luna).

Auclair et al. establecieron un criterio histológico y reproducible, el cual se aplicó en nuestro trabajo. De acuerdo a ellos las características que indican el comportamiento agresivo de un $\mathrm{CME}$ son un componente quístico que ocupa menos del $20 \%$ del área tumoral, cuatro o más figuras de mitosis por diez campos de mayor aumento, invasión neural, necrosis, y anaplasia celular. A cada uno de estos parámetros se le asignó un valor, siendo la suma de todos los puntajes la determinante del grado histológico tumoral (0 a 4 puntos: bajo grado; 5 a 6: intermedio; 7 o más: alto grado).

El grado histológico tumoral se asocia con la sobrevida de los pacientes: la sobrevida de 5 años es del $95 \%$ en tumores de bajo grado de malignidad. Por el contrario, se considera un valor de $40 \%$ para los casos de alto grado de malignidad (Cruz Villca et al., 2007).

Por otro lado, el origen probable de los cristales de colesterol sería la desintegración de eritrocitos dentro de la lesión, ya que se observaron focos hemorrágicos cerca de estos cristales. Llama la atención la presencia de células gigantes de cuerpo extraño, principalmente vecinas a los depósitos de colesterol como pudimos observar en dos casos de CME, uno a nivel de glándulas genianas y otro en el paladar. Algunos autores han sugerido que las células gigantes semejantes a osteoclastos y las de cuerpo extraño tendrían relevancia para estimar el comportamiento tumoral (Gassler et al., 2008). Según Gassler et al. la diferenciación de células gigantes en los tumores salivales es muy rara observándose preferentemente en casos de adenoma pleomórfico localizados en glándulas salivales mayores. También informan que las células gigantes no fueron descritas en tumores primarios de glándulas salivales menores y presentan un caso de CME con depósitos de colesterol y células gigantes de cuerpo extraño en paladar. Estos autores especulan que esta característica corresponde a una entidad tumoral aparte con un pronóstico diferente al de los otros tipos de CME.

La histoquímica convencional no permite reconocer modificaciones en la calidad de las mucinas tumorales en relación a las glándulas salivales normales, por lo que en una próxima investigación estudiaremos con lectinhistoquímica los residuos azúcares terminales y subterminales de los carbohidratos para determinar si se producen cambios ya que la glicosilación aberrante de las proteínas celulares es un rasgo común en el proceso de transformación neoplásica (Samar et al., 2007; Sobral et al., 2010).

Además, continuaremos el presente trabajo con el estudio de los macrófagos asociados al tumor ya que se postula que son marcadores de la progresión tumoral (Shieh et al., 2008).

AVILA, R. E.; SAMAR, M. E.; FONSECA, I.; OLMEDO, L.; ASÍS, O. G. \& FERRARIS, R. Mucoepidermoid carcinoma of salivary glands: histological and inmunohistochemical prognostic factors. Int. J. Morphol., 29(2):455-462, 2011.

SUMMARY: Tumors of the salivary glands represent 2-3\% of head and neck neoplasms. Mucoepidermoid carcinoma (MEC) is the most common epithelial neoplasm in the salivary glands; the biological behavior relates to a histological grade of the tumor (low, intermediate and high grade). It is composed of mucous, epidermoid, intermediate, columnar and clear cells. We undertook this study to analyze the histological grade and proliferative potential as prognostic factors, important to guide the treatment and design recommendations, in a series of 13 patients who were treated for MEC of principal and accessory salivary glands between 1999 and 2009 . Serial cuts of 13 cases of MEC were stained using H/E, Masson, PAS, Dane/Alcian blue and Toluidine blue. Inmunostaining for ki67 were made. Histologically, the tumors were classified as low, intermediate and high grade, according to Auclair et al. system. 8 tumors were located in the parotid, 3 in the palate, 1 in submaxillary and 1 in the genian glands. 3 cases were of high grade, 2 of intermediate grade and 8 of low grade, according to its cytological characteristics, invasive pattern and cell type. In 2 cases of low grade cholesterol clefts, macrophages and foreign-body giant cells were observed. The ki67 expression was important in high grade tumors. The parotid was the most affected gland, followed by the palate and sub maxillary, which agree with the bibliography. The tumoral histological grade was associated with the ki67 expression, relevant prognosis factors in MEC. However, its prognostic value should be validated with clinical studies and survival analyses. Complementary studies to compare carbohydrates of the normal salivary glands must be carried out because subtle alterations in tumor cells glycosylation can influence their biological behavior.

KEY WORDS: Salivary glands; Mucoepidermoid carcinoma; Prognostic factors. 


\section{REFERENCIAS BIBLIOGRAFICAS}

Allon, I.; Vered, M.; Buchner, A.; \& Dayan, D. Stromal differences in salivary gland tumors of the common histopathogenesis but with biological behavior: a study with picrosirius red and polarizing microscopy. Acta Histochem., 108:259-64, 2006.

Ascani, G.; Pieramici, T.; Messi, M.; Lupi, E.; Rubini, C.; \& Balercia P. Salivary glands tumours: a retrospective study of 454 patients. Minerva Stomatol., 55:209-14, 2006.

Auclair, P. L.; Goode, R. K.; \& Ellis, G. L. Mucoepidermoid carcinoma of intraoral salivary glands. Evaluation and application of grading criteria in 143 cases. Cancer, 69(8):2021-30, 1992.

Cruz Villca, M.; Aillón López, H. \& Trujillo, C. Carcinoma mucoepidermoide central: reporte de un caso. Rev. Cuadernos, 52:87-9, 2007.

Dardick, I. Histogenesis and morphogenesis of salivary glands neoplasms. In: Ellis, G. H.; Auclair, P. L. \& Gnepp, D. R. (ed.). Surgical pathology of the salivary glands. Philadelphia, Saunders, 1991.

Ellis, G. L. \& Auclair, P. L. Atlas of tumor pathology: Tumors of the major salivary glands. $4^{\circ}$ serie. Fascículo 9. Washington DC, Armed Forces Institute of Pathology (AFIP), 2008 .

Gassler, N.; Erbe, M.; Caselitz, J. \& Donner, A. S. Mucoepidermoid carcinoma of palatinal glands with exuberant foreign-body giant cell reaction. Pathol. Res. Pract., 204(9):689-91, 2008.

Ito, F. A.; Jorge, J.; Vargas, P. A. \& Lopes, M. A. Histopathological findings of pleomorphic adenomas of salivary glands. Med. Oral Pathol. Oral Cir. Bucal, 14:E57-61, 2009.

Kumar, V. K.; Abbas, A. K. \& Fausto, N. Patologia estructural y funcional. $7^{\circ}$ ed. Madrid, Elsevier, 2005.

Lang, S.; Rotter, N.; Lorenzen, A.; Ihrler, S.; Eckel, R.; Hölzel, D.; Rasp, G.; Wollenberg, G. \& Sommer K. Salivary gland carcinomas. 1- Epidemiology, ethiology, malignancy criteria, prognostic parameters and classification. $H N O$, 53:827-8, 2005.

Leivo, I. Insights into a complex group of neoplastic diseases: advances in histopathologic classification and molecular pathology of salivary gland cancer. Acta Oncol., 45:6628, 2006 .

Licitra, L.; Grandi, C.; Prott, F. J.; Schornagel, J. H.; Bruzzi, P. \& Molinari R. Major and minor salivary glands tumors. Crit. Rev. Oncol. Hematol., 45:215-25, 2003.

Loh, K. S.; Barker, E.; Bruch, G.; O’Sullivan, B.; Brown, D.; Goldstein, D. P.; Gilbert, R. W.; Gullane, D. J. \& Irish, J. C. Prognostic factors in malignancy of the minor salivary glands. Head Neck, 31:58-63, 2009.

Luna, M. A. Salivary mucoepidermoid carcinoma: revisited. Adv. Anat. Pathol., 13:293-307, 2006.

Ozawa, H.; Tomita, T.; Sakamoto, K.; Tagawa, T.; Fujii, R.; Kanzaki, S.; Ogawa, K.; Kameyama, K. \& Fujii, M. Mucoepidermoid carcinoma of the head and neck: clinical analysis of 453 patients. Jpn. J. Clin. Oncol., 38/6:414-8, 2008.

Pérez Ordoñez, B. Selected topics in salivary gland tumour pathology. Current Diagostic Pathology, 9:355-65, 2003.

Pich, A.; Chiusa, L. \& Navone, R. Prognostic relevance of cell proliferation in head and neck tumors. Ann Oncol., 15:1319-29, 2004.

Robinson, R. A. Head and neck pathology. Atlas for histologic and cytologic diagnosis. Philadelphia, Lippincott Williams \& Wilkins, 2010.

Samar, M. E.; Avila, R. E. \& Esteban, R. F. Técnicas histológicas. Fundamentos y aplicaciones. Córdoba, Editorial SeisC, 2004.

Samar, M. E.; Avila, R. E.; Ferraris, R.; Fernández, R. \& Fernández, J. Estudio histoquímico comparativo de las mucinas en células mucosas de glándulas salivales normales y carcinoma mucoepidermoide. Arch. Anat. Costa Rica, 3:47, 2007.

Samar, M. E.; Avila, R. E.; Fonseca, I.; Ferraris, R. \& Fernández, J. Tumores de glándulas salivales: aspectos histopatológicos y clínico-epidemiológicos. Córdoba, Editorial SeisC, 2009.

Shafer, W. \& Levy, B. Tumores de las glándulas salivales. En: Tratado de Patología Bucal. $4^{\circ}$ ed. México, D. F. Interamericana S. A., 2001. pp. 233-61. 
AVILA, R. E.; SAMAR, M. E.; FONSECA, I.; OLMEDO, L.; ASíS, O. G. \& FERRARIS, R. Carcinoma mucoepidermoide de glándulas salivales: factores pronósticos histológicos e inmunohistoquímicos. Int. J. Morphol., 29(2):455-462, 2011.

Shieh, Y. S.; Hung, Y. J.; Hsieh, C. B.; Chen, J. S.; Chou, K. C. \& Liou, S. Y. Tumor-associated macrophage correlated with angiogenesis and progression of mucoepidermoid carcinoma of salivary glands. Ann. Surg. Oncol., 16:751-60, 2008.

Sobral, A. P.; Rego, M. J.; Cavalacanti, C. L.; Carvalho, L. B. Jr. \& Beltrão, E. I. ConA and UEA-I lectin histochemistry of parotid gland mucoepidermoid carcinoma. J. Oral Sci., 52:49-54, 2010.

Speight, P. M. \& Barret, A. W. Salivary glands tumors. Oral Dis., 8:229-40, 2002.

Védrine, P. O.; Coffinet, L.; Ternan, S.; Montagne, K.; Lapeyre, M.; Oberlin, O.; Orbach, D.; Simon, C. \& Sommelet, D. Mucoepidermoid carcinoma of salivary glands in the pediatric age group: 18 clinical cases, including 11 second malignant neoplasms. Head Neck, 28:827-33, 2006.
Dirección para correspondencia:

María Elena Samar

Il Cátedra de Biología Celular, Histología y Embriología.

Facultad de Ciencias Médicas

Cátedra A de Histología y Embriología.

Facultad de Odontología

Universidad Nacional de Córdoba.

Córdoba

ARGENTINA

E-mail: samarcongreso@gmail.com

Recibido : 22-10-2010

Aceptado: 19-02-2011 